\title{
The Modified Objective Function Method for Univex Multiobjective Variational Problems
}

\author{
Tadeusz Antczak ${ }^{1}$ - Anurag Jayswal ${ }^{2}$ - Shalini Jha $^{2}$
}

Received: 5 May 2016 / Accepted: 21 February 2018 / Published online: 24 July 2018

(c) The Author(s) 2018

\begin{abstract}
In this paper, we use the modified objective function method for a class of nonconvex multiobjective variational problems involving univex functions. Under univexity hypotheses, we prove the equivalence between an (weakly) efficient solution of the considered multiobjective variational problem and an (weakly) efficient solution of the associated modified multiobjective variational problem constructed in the modified objective function method.
\end{abstract}

Keyword Multiobjective variational problem, Modified objective function method, Efficient solution, Univexity

Mathematics Subject Classification Primary 65K10; Secondary 90C29 - 90C30 . 90C46 - 90C26

\section{Introduction}

During the last three decades, multiobjective variational problems have been considered in flight control design, control of space structures, industrial process control, impulsive control problems, control of production and inventory, mechanics, eco-

Communicated by Maziar Salahi.

$\bowtie \quad$ Tadeusz Antczak

tadeusz.antczak@wmii.uni.lodz.pl

Anurag Jayswal

anurag_jais123@yahoo.com

Shalini Jha

jhashalini.rash89@gmail.com

1 Faculty of Mathematics and Computer Science, University of Lodz, Banacha 22, 90-238 Lodz, Poland

2 Department of Applied Mathematics, Indian Institute of Technology (Indian School of Mines), Dhanbad, Jharkhand 826004 , India 
nomics, mechanical engineering problems, and other diverse fields. Multiobjective variational programming problems with equality and inequality restrictions were considered by many authors (see, for example, [1,2,4-6,8-18,20-29], and others).

Bector and Husain [10] applied duality method of ordinary vector optimization problem to multiobjective variational problem and obtained duality results for a properly efficient solution under convexity assumptions on the involved functions. Craven [13] obtained the Kuhn-Tucker type necessary conditions for the considered multiobjective variational problem and he proved that they are also sufficient if the objective functions are pseudo-convex and constraints are quasi-convex. Bhatia and Kumar [11] established duality results for Wolfe as well as Mond-Weir-type duals under $\rho$-invexity assumptions and their generalizations for nonconvex multiobjective control problems. Mukherejee and Mishra [22] defined the concept of $V$-invexity for multiobjective variational problems and they established optimality conditions and duality results for multiobjective control problems under (generalized) $V$-invexity assumptions. Thus, they extended the results of Bhatia and Kumar [11] to a wider class of nonconvex multiobjective control problems. In [23], Mukherejee and Rao extended mixed-type duality to the class of multiobjective variational problems and proved duality results under generalized $\rho$-invexity. Bhatia and Mehra [12] extended the concepts of $B$ type I and generalized $B$-type I functions to the continuous case and they used these concepts to establish sufficient optimality conditions and duality results for multiobjective variational programming problems. Nahak and Nanda [25] discussed duality theorems and related efficient solutions to the primal and dual problems for multiobjective variational control problems with $(F, \rho)$-convexity. Reddy and Mukherjee [27] studied duality theorems and related efficient solutions of the primal and dual problems for multiobjective fractional control problems under $(F, \rho)$-convexity. Ahmad and Gulati [2] defined mixed-type duality for multiobjective variational problems, obtaining new optimality results also under $(F, \rho)$-convexity. Using the relationship between an efficient solution of the multiobjective control problem and an optimal solution of the associated scalar control problem, Gramatovici [14] derived the necessary optimality conditions for the multiobjective variational problems with invex functions. Kim and Kim [18] introduced new classes of generalized $V$-type I invex functions for variational problems and they proved a number of sufficiency results and duality theorems using Lagrange multiplier conditions under various types of generalized $V$-type I invexity requirements. Further, under the generalized $V$-type I invexity assumptions and their generalizations, they obtained duality results for Mond-Weirtype duals. Also Hachimi and Aghezzaf [15] obtained several mixed-type duality results for multiobjective variational programming problems, but under a new introduced concept of generalized type I functions. Khazafi and Rueda [16] extended the concept of $V$-univexity type I to multiobjective variational programming problems and they derived various sufficient optimality conditions and mixed-type duality results under generalized $V$-univexity type I conditions. In [17], Khazafi et al. introduced the classes of $(B, \rho)$-type I functions and generalized $(B, \rho)$-type I functions and derived a series of sufficient optimality conditions and mixed-type duality results for multiobjective variational problems. Nahak and Behera [26] used (generalized) $\rho$ - $(\eta, \theta)$ - $B$-type I functions to establish sufficient optimality conditions and duality results for multiobjective variational problems. In [8], Arana-Jimenéz et al. provided 
new pseudoinvexity conditions on the functionals involved in the considered multiobjective variational problem and they proved that all vector Kuhn-Tucker or Fritz John points of a multiobjective variational problem are weakly efficient solutions if and only if these conditions are fulfilled. Recently, Antczak [4] extended the concept of $(\Phi, \rho)$-invexity to the continuous case and, under $(\Phi, \rho)$-invexity hypotheses, he proved sufficient optimality conditions and duality results for a class of nonconvex multiobjective variational problems. Recently, Antczak and Arana-Jiménez [5] proved sufficient optimality conditions and duality results in the sense of Mond-Weir for the considered multiobjective variational control problem under $B$ - $(p, r)$-invexity.

In this paper, we use the modified objective function method introduced by Antczak [3] in the case of differentiable vector optimization problems for solving a nonconvex multiobjective variational problem involving univex functions. In this method, for the considered multiobjective variational problem, its associated multiobjective variational problem with the modified objective function is constructed at an arbitrary but fixed feasible solution. This construction depends heavily on results proved in this paper, which connects an efficient (weakly efficient) solution of the original multiobjective variational problem to an efficient solution (weakly efficient solution) of the modified multiobjective variational problem constructed in the modified objective function method. In general, the multiobjective variational problem with the modified objective function is simpler to solve than the original multiobjective variational problem. Further, there exist also such cases in which the multiobjective variational problem with the modified objective function is convex although the original multiobjective variational problem is nonconvex. These properties are important from a practical point of view. They have been illustrated in the paper by suitable examples of nonconvex multiobjective variational problems.

\section{Preliminaries and Notations}

The following convention for equalities and inequalities will be used in the paper.

For any $x=\left(x_{1}, x_{2}, \ldots, x_{n}\right)^{T}, y=\left(y_{1}, y_{2}, \ldots, y_{n}\right)^{T}$, where ()$^{T}$ denotes for the transpose, we define:

(i) $\quad x=y$ if and only if $x_{i}=y_{i}$ for all $i=1,2, \ldots, n$;

(ii) $x<y$ if and only if $x_{i}<y_{i}$ for all $i=1,2, \ldots, n$;

(iii) $x \leqq y$ if and only if $x_{i} \leqq y_{i}$ for all $i=1,2, \ldots, n$;

(iv) $x \leq y$ if and only if $x \leqq y$ and $x \neq y$.

All vectors will be taken as column vectors.

Let $I=[a, b]$ be a real interval, $A=\{1,2, \ldots, p\}$ and $J=\{1,2, \ldots, m\}$.

In this paper, we assume that $x(t)$ is an $n$-dimensional piecewise smooth function of $t$, and $\dot{x}(t)$ is the derivative of $x(t)$ with respect to $t$ in $[a, b]$.

Denote by $X$ the space of piecewise smooth state functions $x: I \rightarrow R^{n}$ with norm $\|x\|=\|x\|_{\infty}+\|D x\|_{\infty}$, where the differentiation operator $D$ is given by $z=D x \Longleftrightarrow x(t)=x(a)+\int_{a}^{t} z(s) d s$, where $x(a)$ is a given boundary value. 
Therefore, $\frac{d}{d t} \equiv D$ except at discontinuities. For notational simplicity, we write $x(t)$ and $\dot{x}(t)$ as $x$ and $\dot{x}$, respectively.

Let $f: I \times R^{n} \times R^{n} \rightarrow R^{p}$ be a $p$-dimensional function and each of its component is a continuously differentiable real scalar function, $g: I \times R^{n} \times R^{n} \rightarrow R^{m}$ be a continuously differentiable $m$-dimensional function. Here $t$ is the independent variable and $x(t)$ is the state variable. To consider $f^{i}(t, x(t), \dot{x}(t))$, where $x: I \rightarrow R^{n}$ is differentiable with derivative $\dot{x}$, denote the partial derivatives of $f^{i}, i \in A$, with respect to $t, x$ and $\dot{x}$, respectively, by $f_{t}^{i}, f_{x}^{i}, f_{\dot{x}}^{i}$ such that $f_{x}^{i}=\left(\frac{\partial f^{i}}{\partial x_{1}}, \ldots, \frac{\partial f^{i}}{\partial x_{n}}\right)$ and $f_{\dot{x}}^{i}=\left(\frac{\partial f^{i}}{\partial \dot{x}_{1}}, \ldots, \frac{\partial f^{i}}{\partial \dot{x}_{n}}\right)$. Similarly, the partial derivatives of the vector function $g$ can be written, using matrices with $m$ rows instead of one. Further, we denote by diag $z$ a $p \times p$-dimensional matrix as follows:

$$
\operatorname{diag} z=\left[\begin{array}{cccc}
z_{1} & 0 & \ldots & 0 \\
0 & z_{2} & \ldots & \vdots \\
\vdots & & \ddots & 0 \\
0 & \ldots & 0 & z_{p}
\end{array}\right]
$$

In the past few years, extensive literature relative to the other families of more general functions to substitute the convex functions in the optimization theory has grown immensely. One of such classes of generalized convex functions is the class of univex functions, introduced by Bector et al. [7] for scalar optimization problems.

Let $f: I \times R^{n} \times R^{n} \rightarrow R^{p}$ be a continuously differentiable functional with respect to each of their arguments and $\bar{x} \in X$. The following definition introduces the concept of univexity for the functional $f$.

Definition 2.1 If there exist functions $\Phi_{f}=\left(\Phi_{f^{1}}, \ldots, \Phi_{f^{p}}\right): R^{p} \rightarrow R^{p}, b_{f}=$ $\left(b_{f^{1}}, \ldots, b_{f^{p}}\right): X \times X \rightarrow R_{+}^{p}$, where $b_{f^{i}}: X \times X \rightarrow R_{+} \backslash\{0\}, i=1, \ldots, p$, $\eta: I \times R^{n} \times R^{n} \rightarrow R^{n}$ such that the inequality

$$
\begin{aligned}
& \operatorname{diag} b_{f}(x, \bar{x}) \Phi_{f}\left(\int_{a}^{b} f(t, x, \dot{x}) d t-\int_{a}^{b} f(t, \bar{x}, \dot{\bar{x}}) d t\right) \\
& \geqq(>) \int_{a}^{b}\left\{\eta(t, x, \bar{x})^{T} f_{x}(t, \bar{x}, \dot{\bar{x}})+\frac{d}{d t}\left(\eta(t, x, \bar{x})^{T}\right) f_{\dot{x}}(t, \bar{x}, \dot{\bar{x}})\right\} d t
\end{aligned}
$$

holds for all $x \in X,(x \neq \bar{x})$, then the functional $f$ is said to be (strictly) univex at $\bar{x}$ with respect to $\Phi, b, \eta$. If (1) is satisfied for every $\bar{x} \in X$, then $f$ is said to be (strictly) univex on $X$.

Equivalently, the inequality (1), defining the concept of univexity, can be written as follows: 


$$
\begin{aligned}
& b_{f^{i}}(x, \bar{x}) \Phi_{f^{i}}\left(\int_{a}^{b} f^{i}(t, x, \dot{x}) d t-\int_{a}^{b} f^{i}(t, \bar{x}, \dot{\bar{x}}) d t\right) \\
& \geqq(>) \int_{a}^{b}\left\{\eta(t, x, \bar{x})^{T} f_{x}^{i}(t, \bar{x}, \dot{\bar{x}})+\frac{d}{d t}\left(\eta(t, x, \bar{x})^{T}\right) f_{\dot{x}}^{i}(t, \bar{x}, \dot{\bar{x}})\right\} d t, i \in A .
\end{aligned}
$$

Remark 2.2 Note that the concept of univexity generalizes some generalized convexity notions previously defined in the literature for multiobjective variational problems. Indeed, in the case when $\Phi_{f^{i}}=I d_{R^{p}}$, where $I d_{R^{p}}$ is the identity in $R^{p}$, then we obtain the definition of a $b$-invex function in a continuous case (see [1]). Moreover, if $b_{f^{i}}(x, \bar{x}) \equiv 1, i=1, \ldots, p$, for all $x, \bar{x} \in X$, then we obtain the definition of an invex function in a continuous case (see, for example, [1]).

In the multiobjective variational control problem, under given conditions, the state vector $x(t)$ is brought from specified initial state $x(a)=\alpha$ to some specified final state $x(b)=\beta$ in such a way to minimize a given functional. A more precise mathematical formulation is given in the following multiobjective variational programming problem:

$$
\begin{aligned}
& V \text {-Minimize } \int_{a}^{b} f(t, x(t), \dot{x}(t)) \mathrm{d} t \\
& \quad=\left(\int_{a}^{b} f^{1}(t, x(t), \dot{x}(t)) \mathrm{d} t, \ldots, \int_{a}^{b} f^{p}(t, x(t), \dot{x}(t)) \mathrm{d} t\right) \\
& \text { subject to } g(t, x(t), \dot{x}(t)) \leqq 0, \quad t \in I, \\
& x(a)=\alpha, \quad(b)=\beta,
\end{aligned}
$$

where $f=\left(f^{1}, \ldots, f^{p}\right): I \times R^{n} \times R^{n} \rightarrow R^{p}$ and $g=\left(g^{1}, \ldots, g^{m}\right): I \times R^{n} \times R^{n} \rightarrow$ $R^{m}$ are continuously differentiable functions with respect to each of their arguments.

Let $S$ denote the set of all feasible solutions for the considered multiobjective variational programming problem (MVP), i.e.,

$$
S=\left\{x \in R^{n}: g(t, x(t), \dot{x}(t)) \leqq 0, \forall t \in I, x(a)=\alpha, x(b)=\beta\right\} .
$$

Definition 2.3 A feasible solution $\bar{x}$ of the considered multiobjective variational programming problem (MVP) is said to be weakly efficient of (MVP) if there exists no other $x \in S$ such that

$$
\int_{a}^{b} f(t, x, \dot{x}) \mathrm{d} t<\int_{a}^{b} f(t, \bar{x}, \dot{\bar{x}}) \mathrm{d},
$$


that is, there exists no other $x \in S$ such that

$$
\int_{a}^{b} f^{i}(t, x, \dot{x}) \mathrm{d} t<\int_{a}^{b} f^{i}(t, \bar{x}, \bar{x}) \mathrm{d} t, \quad \forall i \in A .
$$

Definition 2.4 A feasible solution $\bar{x}$ of the considered multiobjective variational programming problem (MVP) is said to be efficient of (MVP) if there exists no other $x \in S$ such that

$$
\int_{a}^{b} f(t, x, \dot{x}) \mathrm{d} t \leq \int_{a}^{b} f(t, \bar{x}, \dot{\bar{x}}) \mathrm{d} t,
$$

that is, there exists no other $x \in S$ such that

$$
\begin{aligned}
& \int_{a}^{b} f^{i}(t, x, \dot{x}) \mathrm{d} t \leqq \int_{a}^{b} f^{i}(t, \bar{x}, \dot{\bar{x}}) \mathrm{d} t, \quad \forall i \in A, \\
& \int_{a}^{b} f^{i^{*}}(t, x, \dot{x}) \mathrm{d} t<\int_{a}^{b} f^{i^{*}}(t, \bar{x}, \dot{\bar{x}}) \mathrm{d} t \text { for at least one } i^{*} \in A .
\end{aligned}
$$

In order to prove sufficient optimality conditions for the considered multiobjective variational programming problem (MVP), we give the Karush-Kuhn-Tucker necessary optimality conditions for such vector optimization problems (see, for instance, $[5,6,12,19,26])$.

Theorem 2.5 Let $\bar{x} \in S$ be a normal efficient solution of the considered multiobjective variational programming problem $(M V P)$ at which the Kuhn-Tucker constraint qualification be satisfied. Then there exist $\bar{\lambda} \in R^{p}$ and the piecewise smooth function $\bar{\xi}(\cdot): I \rightarrow R^{m}$ such that

$$
\begin{gathered}
\bar{\lambda}^{T} f_{x}(t, \bar{x}, \dot{\bar{x}})+\bar{\xi}(t)^{T} g_{x}(t, \bar{x}, \dot{\bar{x}}) \\
=\frac{d}{\mathrm{~d} t}\left[\bar{\lambda}^{T} f_{\dot{x}}(t, \bar{x}, \dot{\bar{x}})+\bar{\xi}(t)^{T} g_{\dot{x}}(t, \bar{x}, \dot{\bar{x}})\right], t \in I, \\
\bar{\xi}(t)^{T} g(t, \bar{x}, \dot{\bar{x}}) \mathrm{d} t=0, t \in I, \\
\bar{\lambda} \geq 0, \quad \bar{\lambda}^{T} e=1, \quad \bar{\xi}(t) \geqq 0 .
\end{gathered}
$$

For notational convenience, we use $\xi$ for $\xi(t)$. 
Remark 2.6 [12] We shall use the following property to prove the main results in the paper.

Let $h: I \times R^{n} \times R^{n} \rightarrow R$ be a continuously differentiable function with respect to each of its arguments and let $\eta: I \times R^{n} \times R^{n} \rightarrow R^{n}$ satisfy the condition $\eta(t, x, x)=0$. Let $x, u: I \rightarrow R^{n}$ be differentiable functions with $x(a)=u(a)=\alpha$ and $x(b)=u(b)=\beta$. Then,

$$
\int_{a}^{b} \frac{d}{\mathrm{~d} t}(\eta(t, x, u)) h_{\dot{x}}(t, u, \dot{u}) \mathrm{d} t=-\int_{a}^{b} \eta(t, x, u)\left(\frac{d}{\mathrm{~d} t} h_{\dot{x}}(t, u, \dot{u})\right) \mathrm{d} t .
$$

\section{Multiobjective Variational Problem with the Modified Objective Function}

Let $\bar{x}$ be an arbitrary feasible solution of the considered multiobjective variational programming problem (MVP) and the vector-valued function $\eta: I \times R^{n} \times R^{n} \rightarrow R^{n}$ be given. We now use the modified objective function method for solving the considered nonconvex multiobjective variational programming problem (MVP). Therefore, at the given feasible point $\bar{x}$, we construct the multiobjective variational programming problem $\left(\operatorname{MVP}_{\eta}(\bar{x})\right)$ with the modified objective function associated with the original multiobjective variational programming problem (MVP) as follows:

$$
\begin{aligned}
& \qquad \int_{a}^{V \text {-Minimize }} f^{1}(t, \bar{x}(t), \bar{x}(t)) \mathrm{d} t+\int_{a}^{b}\left\{\eta(t, x(t), \bar{x}(t))^{T} f_{x}^{1}(t, \bar{x}(t), \dot{\bar{x}}(t))\right. \\
& \left.\quad+\frac{d}{\mathrm{~d} t}\left(\eta(t, x(t), \bar{x}(t))^{T}\right) f_{\dot{x}}^{1}(t, \bar{x}(t), \bar{x}(t))\right\} \mathrm{d} t, \ldots, \\
& \int_{a}^{b} f^{p}(t, \bar{x}(t), \dot{x}(t)) \mathrm{d} t+\int_{a}^{b}\left\{\eta(t, x(t), \bar{x}(t))^{T} f_{x}^{p}(t, \bar{x}(t), \dot{\bar{x}}(t))\right. \\
& \left.\left.\quad+\frac{d}{\mathrm{~d} t}\left(\eta(t, x(t), \bar{x}(t))^{T}\right) f_{\dot{x}}^{p}(t, \bar{x}(t), \bar{x}(t))\right\} \mathrm{d} t\right) \quad\left(M V P_{\eta}\right. \\
& \text { subject to } g(t, x(t), \dot{x}(t)) \leqq 0, \quad t \in I
\end{aligned}
$$$$
\left(M V P_{\eta}(\bar{x})\right)
$$$$
x(a)=\alpha \quad, \quad x(b)=\beta,
$$

where $f=\left(f^{1}, \ldots, f^{p}\right): I \times R^{n} \times R^{n} \rightarrow R^{p}$ and $g=\left(g^{1}, \ldots, g^{m}\right): I \times R^{n} \times R^{n} \rightarrow$ $R^{m}$ are continuously differentiable functions with respect to each of their arguments.

Now, we prove the equivalence between an efficient solution (weakly efficient solution) of the original multiobjective variational problem and an efficient solution 
(weakly efficient solution) of its associated modified multiobjective variational problem constructed in the modified objective function method.

Theorem 3.1 Let $\bar{x}$ be a feasible solution of the considered multiobjective variational programming problem (MVP) and the Karush-Kuhn-Tuckernecessaryoptimality conditions (2.3)-(2.5) be satisfied at this point with $\bar{\lambda} \in R^{p}$ and the piecewise smooth function $\bar{\xi}(\cdot): I \rightarrow R^{m}$. Further, assume that $\bar{\xi}(t)^{T} g(t, \cdot, \cdot)$ is univex at $\bar{x}$ on $S$ with respect to $\Phi_{g}, b_{g}, \eta$, where $a \leqq 0 \Longrightarrow \Phi_{g}(a) \leqq 0$ and $\eta(t, \bar{x}, \bar{x})=0$. If $\bar{\lambda}>0$, then $\bar{x}$ is an efficient solution of the multiobjective variational programming problem with the modified objective function $\left(M V P_{\eta}(\bar{x})\right)$ associated with the original multiobjective variational problem (MVP).

Proof Suppose, contrary to the result, that $\bar{x}$ is not an efficient solution of the multiobjective variational programming problem $\left(\operatorname{MVP}_{\eta}(\bar{x})\right)$ with the modified objective function associated with the original multiobjective variational problem (MVP). Then, there exists $\tilde{x} \in S$ such that

$$
\begin{aligned}
& \int_{a}^{b}\left\{\eta(t, \tilde{x}, \bar{x})^{T} f_{x}^{i}(t, \bar{x}, \dot{\bar{x}})+\frac{d}{\mathrm{~d} t}\left(\eta(t, \tilde{x}, \bar{x})^{T}\right) f_{\dot{x}}^{i}(t, \bar{x}, \dot{\bar{x}})\right\} \mathrm{d} t \leqq \\
& \int_{a}^{b}\left\{\eta(t, \bar{x}, \bar{x})^{T} f_{x}^{i}(t, \bar{x}, \dot{\bar{x}})+\frac{d}{\mathrm{~d} t}\left(\eta(t, \bar{x}, \bar{x})^{T}\right) f_{\dot{x}}^{i}(t, \bar{x}, \dot{\bar{x}})\right\} \mathrm{d} t, \forall i \in A \\
& \int_{a}^{b}\left\{\eta(t, \tilde{x}, \bar{x})^{T} f_{\dot{x}}^{i^{*}}(t, \bar{x}, \dot{\bar{x}})+\frac{d}{\mathrm{~d} t}\left(\eta(t, \tilde{x}, \bar{x})^{T}\right) f_{\dot{x}}^{i^{*}}(t, \bar{x}, \dot{\bar{x}})\right\} \mathrm{d} t< \\
& \int_{a}^{b}\left\{\eta(t, \bar{x}, \bar{x})^{T} f_{x}^{i^{*}}(t, \bar{x}, \dot{\bar{x}})+\frac{d}{\mathrm{~d} t}\left(\eta(t, \bar{x}, \bar{x})^{T}\right) f_{\dot{x}}^{i^{*}}(t, \bar{x}, \dot{\bar{x}})\right\} \mathrm{d} t \\
& \text { for at least one } i^{*} \in A .
\end{aligned}
$$

Since $\eta(t, \bar{x}(t), \bar{x}(t))=0$, inequalities (3.1) and (3.2) yield, respectively,

$$
\begin{gathered}
\int_{a}^{b}\left\{\eta(t, \tilde{x}, \bar{x})^{T} f_{x}^{i}(t, \bar{x}, \dot{\bar{x}})+\frac{d}{\mathrm{~d} t}\left(\eta(t, \tilde{x}, \bar{x})^{T}\right) f_{\dot{x}}^{i}(t, \bar{x}, \dot{\bar{x}})\right\} \mathrm{d} t \leqq 0, \forall i \in A \\
\int_{a}^{b}\left\{\eta(t, \tilde{x}, \bar{x})^{T} f_{x}^{i^{*}}(t, \bar{x}, \dot{\bar{x}})+\frac{d}{\mathrm{~d} t}\left(\eta(t, \tilde{x}, \bar{x})^{T}\right) f_{\dot{x}}^{i^{*}}(t, \bar{x}, \dot{\bar{x}})\right\} \mathrm{d} t<0
\end{gathered}
$$

for at least one $i^{*} \in A$. (3.4) 
By assumption, $\bar{\lambda}>0$. Hence, (3.3) and (3.4) imply

$$
\int_{a}^{b}\left\{\eta(t, \tilde{x}, \bar{x})^{T} \bar{\lambda}^{T} f_{x}(t, \bar{x}, \dot{\bar{x}})+\frac{d}{\mathrm{~d} t}\left(\eta(t, \tilde{x}, \bar{x})^{T}\right) \bar{\lambda}^{T} f_{\dot{x}}(t, \bar{x}, \dot{\bar{x}})\right\} \mathrm{d} t<0 .
$$

By (2.6), inequality (3.5) gives

$$
\int_{a}^{b}\left\{\eta(t, \tilde{x}, \bar{x})^{T}\left[\bar{\lambda}^{T} f_{x}(t, \bar{x}, \dot{x})-\bar{\lambda}^{T} \frac{d}{\mathrm{~d} t}\left(f_{\dot{x}}(t, \bar{x}, \dot{\bar{x}})\right)\right]\right\} \mathrm{d} t<0 .
$$

Now, using $\tilde{x}, \bar{x} \in S$ together with the necessary optimality conditions (2.4) and (2.5), we get

$$
\int_{a}^{b} \bar{\xi}(t)^{T} g(t, \tilde{x}, \dot{\tilde{x}}) \mathrm{d} t-\int_{a}^{b} \bar{\xi}(t)^{T} g(t, \bar{x}, \dot{\bar{x}}) \mathrm{d} t \leqq 0 .
$$

Hence, by hypothesis $a \leqq 0 \Longrightarrow \Phi_{g}(a) \leqq 0$, the above inequality implies

$$
\Phi_{g}\left(\int_{a}^{b} \bar{\xi}(t)^{T} g(t, \tilde{x}, \dot{\tilde{x}}) \mathrm{d} t-\int_{a}^{b} \bar{\xi}(t)^{T} g(t, \bar{x}, \dot{\bar{x}}) \mathrm{d} t\right) \leqq 0
$$

Since $b_{g}(\tilde{x}, \bar{x})>0,(3.7)$ gives

$$
b_{g}(\tilde{x}, \bar{x}) \Phi_{g}\left(\int_{a}^{b} \bar{\xi}(t)^{T} g(t, \tilde{x}, \dot{\tilde{x}}) \mathrm{d} t-\int_{a}^{b} \bar{\xi}(t)^{T} g(t, \bar{x}, \dot{\bar{x}}) \mathrm{d} t\right) \leqq 0 .
$$

Hence, by Definition 2.1, (3.8) implies

$$
\int_{a}^{b}\left\{\eta(t, \tilde{x}, \bar{x})^{T} \bar{\xi}(t)^{T} g_{x}(t, \bar{x}, \dot{\bar{x}})+\frac{d}{\mathrm{~d} t}\left(\eta(t, \tilde{x}, \bar{x})^{T}\right) \bar{\xi}(t)^{T} g_{\dot{x}}(t, \bar{x}, \dot{\bar{x}})\right\} \mathrm{d} t \leqq 0 .
$$

By (2.6), the above inequality (3.9) gives

$$
\int_{a}^{b}\left\{\eta(t, \tilde{x}, \bar{x})^{T} \bar{\xi}(t)^{T} g_{x}(t, \bar{x}, \dot{x})-\eta(t, \tilde{x}, \bar{x})^{T} \frac{d}{\mathrm{~d} t}\left(\bar{\xi}(t)^{T} g_{\dot{x}}(t, \bar{x}, \dot{x})\right)\right\} \mathrm{d} t \leqq 0 .
$$


Combining (3.6) and (3.10), we obtain that the following inequality:

$$
\begin{aligned}
\int_{a}^{b} \eta(t, \tilde{x}, \bar{x})^{T} & {\left[\bar{\lambda}^{T} f_{x}(t, \bar{x}, \dot{\bar{x}})+\bar{\xi}(t)^{T} g_{x}(t, \bar{x}, \dot{\bar{x}})\right.} \\
- & \left.\frac{d}{\mathrm{~d} t}\left(\bar{\lambda}^{T} f_{\dot{x}}(t, \bar{x}, \dot{\bar{x}})+\bar{\xi}(t)^{T} g_{\dot{x}}(t, \bar{x}, \dot{\bar{x}})\right)\right] \mathrm{d} t<0
\end{aligned}
$$

holds, which contradicts the necessary optimality conditions (2.3). This completes the proof of this theorem.

Corollary 3.2 Let $\bar{x}$ be an efficient solution of the considered multiobjective variational programming problem (MVP) and the Karush-Kuhn-Tuckernecessaryoptimalityconditions (2.3)-(2.5) be satisfied at this point with $\bar{\lambda} \in R^{p}$ and the piecewise smooth function $\bar{\xi}(\cdot): I \rightarrow R^{m}$. Further, assume that $\bar{\xi}(t)^{T} g(t, \cdot, \cdot)$ is univex at $\bar{x}$ on $S$ with respect to $\Phi_{g}, b_{g}, \eta$, where $a \leqq 0 \Longrightarrow \Phi_{g}(a) \leqq 0$ and $\eta(t, \bar{x}, \bar{x})=0$. If $\bar{\lambda}>0$, then $\bar{x}$ is an efficient solution of the multiobjective variational programming problem $\left(\operatorname{MVP}_{\eta}(\bar{x})\right)$ with the modified objective function associated with the original variational programming problem (MVP).

In order to prove that a feasible solution $\bar{x}$ is weakly efficient in problem (MVP), the assumption $\bar{\lambda}>0$ can be omitted.

Corollary 3.3 Let $\bar{x}$ be a weakly efficient solution of the considered multiobjective variational programming problem (MVP) and the Karush-Kuhn-Tucker conditions (2.3)-(2.5) be satisfied at this point with $\bar{\lambda} \in R^{p}$ and the piecewise smooth function $\bar{\xi}(\cdot): I \rightarrow R^{m}$. Further, assume that $\bar{\xi}(t)^{T} g(t, \cdot, \cdot)$ is univex at $\bar{x}$ on $S$ with respect to $\Phi_{g}, b_{g}, \eta$, where $a \leqq 0 \Longrightarrow \Phi_{g}(a) \leqq 0$ and $\eta(t, \bar{x}, \bar{x})=0$. Then $\bar{x}$ is a weakly efficient solution of the multiobjective variational programming problem $\left(\operatorname{MVP}_{\eta}(\bar{x})\right)$ with the modified objective function associated with the original variational programming problem (MVP).

Remark 3.4 Note that we have established the results in Theorem 3.1 and Corollaries 3.2 and 3.3 without any univexity assumption imposed on the objective function.

Now, we prove the converse results to those ones established above.

Theorem 3.5 Let $\bar{x}$ be an efficient solution of the multiobjective variational programming problem $\left(\operatorname{MVP}_{\eta}(\bar{x})\right)$ with the modified objective function. Further, assume that $f^{i}(t, \cdot, \cdot), i=1, \ldots, p$, is strictly univex at $\bar{x}$ on $S$ with respect to $\Phi_{f^{i}}, b_{f^{i}}, \eta$, where $a \leqq 0 \Longrightarrow \Phi_{f^{i}}(a) \leqq 0, i \in I$, and, moreover, $\eta(t, \bar{x}, \bar{x})=0$. Then $\bar{x}$ is an efficient solution of the considered multiobjective variational problem (MVP).

Proof Suppose, contrary to the result, that $\bar{x}$ is not a weakly efficient solution of the considered multiobjective variational programming problem (MVP). Then, there exist $\tilde{x} \in S$ and $\tilde{x} \neq \bar{x}$ such that

$$
\int_{a}^{b} f(t, \tilde{x}, \dot{\tilde{x}}) \mathrm{d} t \leq \int_{a}^{b} f(t, \bar{x}, \dot{\bar{x}}) \mathrm{d} t .
$$


Thus,

$$
\begin{gathered}
\int_{a}^{b} f^{i}(t, \tilde{x}, \dot{\tilde{x}}) \mathrm{d} t \leqq \int_{a}^{b} f^{i}(t, \bar{x}, \dot{\bar{x}}) \mathrm{d} t, \quad i \in A \\
\int_{a}^{b} f^{i^{*}}(t, \tilde{x}, \dot{\tilde{x}}) \mathrm{d} t<\int_{a}^{b} f^{i^{*}}(t, \bar{x}, \bar{x}) \mathrm{d} t \text { for at least on } i^{*} \in A .
\end{gathered}
$$

Then, by hypothesis imposed on $\Phi_{f}$, inequalities (3.11) and (3.12) yield, respectively,

$$
\Phi_{f^{i}}\left(\int_{a}^{b} f^{i}(t, \tilde{x}, \dot{\tilde{x}}) \mathrm{d} t-\int_{a}^{b} f^{i}(t, \bar{x}, \dot{\bar{x}}) \mathrm{d} t\right) \leqq 0, \quad i \in A
$$

Since each function $f^{i}(t, \cdot, \cdot), i=1, \ldots, p$, is strictly univex at $\bar{x}$ on $S$ with respect to $\Phi_{f^{i}}, b_{f^{i}}, \eta$, by Definition 2.1, the following inequalities

$$
\begin{aligned}
& b_{f^{i}}(x, \bar{x}) \Phi_{f^{i}}\left(\int_{a}^{b} f^{i}(t, x, \dot{x}) \mathrm{d} t-\int_{a}^{b} f^{i}(t, \bar{x}, \dot{x})\right) \mathrm{d} t \\
& \quad>\int_{a}^{b}\left\{\eta(t, x, \bar{x})^{T} f_{x}^{i}(t, \bar{x}, \dot{\bar{x}})+\frac{d}{\mathrm{~d} t}\left(\eta(t, x, \bar{x})^{T}\right) f_{\dot{x}}^{i}(t, \bar{x}, \dot{\bar{x}})\right\} \mathrm{d} t, i \in A
\end{aligned}
$$

hold for all $x \in S,(x \neq \bar{x})$. Therefore, the inequalities above are also satisfied for $x=\tilde{x} \in S$. Thus,

$$
\begin{aligned}
& b_{f^{i}}(\tilde{x}, \bar{x}) \Phi_{f^{i}}\left(\int_{a}^{b} f^{i}(t, \tilde{x}, \dot{\tilde{x}}) \mathrm{d} t-\int_{a}^{b} f^{i}(t, \bar{x}, \dot{\bar{x}})\right) \mathrm{d} t \\
& \quad>\int_{a}^{b}\left\{\eta(t, \tilde{x}, \bar{x})^{T} f_{x}^{i}(t, \bar{x}, \dot{\bar{x}})+\frac{d}{\mathrm{~d} t}\left(\eta(t, \tilde{x}, \bar{x})^{T}\right) f_{\dot{x}}^{i}(t, \bar{x}, \dot{\bar{x}})\right\} \mathrm{d} t, i \in A .
\end{aligned}
$$

Combining (3.13) and (3.14), we get

$$
\int_{a}^{b}\left\{\eta(t, \tilde{x}, \bar{x})^{T} f_{x}^{i}(t, \bar{x}, \dot{x})+\frac{d}{\mathrm{~d} t}\left(\eta(t, \tilde{x}, \bar{x})^{T}\right) f_{\dot{x}}^{i}(t, \bar{x}, \dot{\bar{x}})\right\} \mathrm{d} t<0, i \in A
$$


By assumption, $\eta(t, \bar{x}, \bar{x})=0$. Hence, (3.15) implies that the following inequalities

$$
\begin{aligned}
& \int_{a}^{b} f^{i}(t, \bar{x}, \bar{x}) \mathrm{d} t+\int_{a}^{b}\left\{\eta(t, \tilde{x}, \bar{x})^{T} f_{x}^{i}(t, \bar{x}, \dot{\bar{x}})+\frac{d}{\mathrm{~d} t}\left(\eta(t, \tilde{x}, \bar{x})^{T}\right)^{T} f_{\dot{x}}^{i}(t, \bar{x}, \dot{\bar{x}})\right\} \mathrm{d} t \\
& <\int_{a}^{b} f^{i}(t, \bar{x}, \dot{\bar{x}}) \mathrm{d} t+\int_{a}^{b}\left\{\eta(t, \bar{x}, \bar{x})^{T} f_{x}^{i}(t, \bar{x}, \dot{\bar{x}})+\frac{d}{\mathrm{~d} t}\left(\eta(t, \bar{x}, \bar{x})^{T}\right) f_{\dot{x}}^{i}(t, \bar{x}, \dot{\bar{x}})\right\} \mathrm{d} t
\end{aligned}
$$

hold, contradicting the efficiency of $\bar{x}$ for the multiobjective variational programming problem $\left(\operatorname{MVP}_{\eta}(\bar{x})\right)$ with the modified objective function associated with the original multiobjective variational problem (MVP). This completes the proof of this theorem.

Remark 3.6 Note that we have established the result in Theorem 3.5 without any univexity assumption imposed on the constraint function.

If we assume weaker univexity hypothesis imposed on the objective function, then we are in a position to obtain the following result.

Theorem 3.7 Let $\bar{x}$ be a weakly efficient solution of the multiobjective variational problem $\left(\operatorname{MVP}_{\eta}(\bar{x})\right)$ with the modified objective function. Further, assume that each objective function $f^{i}(t, \cdot, \cdot), i=1, \ldots, p$, is univex at $\bar{x}$ on $S$ with respect to $\Phi_{f^{i}}, b_{f^{i}}$, $\eta$, where $a<0 \Longrightarrow \Phi_{f^{i}}(a)<0, i \in I$, and, moreover, $\eta(t, \bar{x}, \bar{x})=0$. Then $\bar{x}$ is a weakly efficient solution of the considered multiobjective variational problem (MVP).

By Corollary 3.2 and Theorem 3.5, it follows the equivalence between an efficient solution of the original multiobjective variational problem and an efficient solution of its associated modified multiobjective variational problem constructed in the modified objective function method.

Theorem 3.8 Let all hypotheses of Theorems 3.1 and 3.5 be fulfilled. Then $\bar{x} \in S$ is an efficient solution of the original multiobjective variational problem (MVP) if and only if it is an efficient solution of its associated modified multiobjective variational problem constructed in the modified objective function method.

The similar result is true by Corollary 3.3 and Theorem 3.7:

Theorem 3.9 Let all hypotheses of Corollary 3.3 and Theorem 3.7 be fulfilled. Then a feasible solution $\bar{x}$ is a weakly efficient solution of the original multiobjective variational problem (MVP) if and only if it is a weakly efficient solution of its associated modified multiobjective variational problem constructed in the modified objective function method.

Now, we give an example of a nonconvex multiobjective variational problem to illustrate the result established in the paper. 
Example 3.10 Let $I=[0,1]$. Consider the following nonconvex multiobjective variational problem:

$$
\begin{aligned}
V \text {-Minimize } & \int_{0}^{1} f(t, x, \dot{x}) \mathrm{d} t=\left(\int_{0}^{1}\left(1+t+x-x^{2}+\sin ^{2} x\right) \mathrm{d} t,\right. \\
& \left.\int_{0}^{1}\left(t+x e^{x}\right) \mathrm{d} t\right) \\
& \text { subject to } g(t, x, \dot{x})=x^{2}-x \leqq 0, \quad t \in I \\
& x(0)=0, x(1)=0 .
\end{aligned}
$$

The set of all feasible solutions of the considered multiobjective variational problem (MVP1) is given by $S=\{x \in R: x(0)=0, x(1)=0$ and $0 \leqq x(t) \leqq 1$ for each $t \in I\}$ and, moreover, $\bar{x}(t)=0$ is feasible for the problem (MVP1). Further, note that $\bar{\lambda}=\left(\bar{\lambda}_{1}, \bar{\lambda}_{2}\right)=\left(\frac{1}{2}, \frac{1}{2}\right)$ and $\bar{\xi}(t)=1$ verify Theorem 2.5 .

We set

$$
\begin{aligned}
\Phi_{f_{1}}(a) & =e^{a}-1, \Phi_{f_{2}}(a)=a, \Phi_{g}(a)=a, \\
b_{f_{1}}(x, \bar{x}) & =1, b_{f_{2}}(x, \bar{x})=1, b_{g}(x, \bar{x})=1
\end{aligned}
$$

and, moreover, $\eta: I \times R \times R \mapsto R$ be defined as

$$
\eta(t, x, \bar{x})=x-x^{2}-\bar{x}-\bar{x}^{2} .
$$

Hence, it can be established by Definition 2.1 that $f^{i}(t, \cdot, \cdot), i=1,2$, is strictly univex at $\bar{x}$ on $S$ with respect to $\Phi_{f^{i}}, b_{f^{i}}$, and $\eta$ and $\bar{\xi}(t)^{T} g(t, \cdot, \cdot)$ is univex at $\bar{x}$ on $S$ with respect to $\Phi_{g}, b_{g}$ and $\eta$. Using the approach analyzed in the paper, we construct the following multiobjective variational problem $\left(\operatorname{MVP}_{\eta}(\bar{x})\right)$ with the modified objective function as follows:

$$
\begin{gathered}
V \text {-Minimize } \\
\left(\int_{0}^{1}\left(1+t+x-x^{2}\right) \mathrm{d} t, \int_{0}^{1}\left(t+x-x^{2}\right) \mathrm{d} t\right) \\
\text { subject to } g(t, x, \dot{x})=x^{2}-x \leqq 0, \quad t \in I \\
x(0)=0, x(1)=0 .
\end{gathered}
$$

Since all hypotheses of Theorem 3.1 are fulfilled, we conclude that $\bar{x}(t)=0$ is an efficient solution of the multiobjective variational problem $\left(\operatorname{MVP} 1_{\eta}(\bar{x})\right)$ with the modified objective function. Further, all hypotheses of Theorem 3.5 are also satisfied at $\bar{x}=0$. Therefore, $\bar{x}(t)=0$, being efficient in the multiobjective variational problem $\left(\mathrm{MVP}_{\eta}(\bar{x})\right)$, it is also efficient for the original multiobjective variational problem (MVP1). Note, moreover, that this result cannot be proved under convexity hypotheses since the functions constituting the multiobjective variational problem (MVP1) are not convex on $S$.

Now, we give an example of a nonconvex multiobjective variational problem to illustrate one of the properties of the approach analyzed in the paper. Namely, for some 
nonlinear nonconvex multiobjective variational problems, multiobjective variational problems with the modified objective function constructed in the analyzed approach may be linear.

Example 3.11 Let $I=[0,1]$. Consider the following nonconvex multiobjective variational problem:

$$
\begin{aligned}
& V \text {-Minimize } \int_{0}^{1} f(t, x, \dot{x}) \mathrm{d} t=\left(\int_{0}^{1}\left(t x^{3}+\frac{1}{2} x\right) \mathrm{d} t, \int_{0}^{1}\left(\sin x+x-\frac{3}{4} x^{2}\right) \mathrm{d} t\right) \\
& \text { subject to } g_{1}(t, x, \dot{x})=-x \leqq 0, \quad t \in I \\
& \quad g_{2}(t, x, \dot{x})=x-1 \leqq 0, \quad t \in I, \\
& \quad x(0)=0, x(1)=0 .
\end{aligned}
$$

The set of all feasible solutions of problem (MVP2) is given by $S=\{x \in R: x(0)=$ $0, x(1)=0$ and $0 \leqq x(t) \leqq 1$ for each $t \in I\}$ and $\bar{x}(t)=0$. Further, note that $\bar{\lambda}=\left(\bar{\lambda}_{1}, \bar{\lambda}_{2}\right)=\left(\frac{1}{2}, \frac{1}{2}\right)$ and $\bar{\xi}(t)=\left(\frac{5}{4}, 0\right)$ verify Theorem 2.5 .

We set

$$
\begin{gathered}
\Phi_{f_{1}}(a)=a, \Phi_{f_{2}}(a)=a, \Phi_{g}(a)=a, \\
b_{f_{1}}(x, \bar{x})=\frac{1}{2}, b_{f_{2}}(x, \bar{x})=2, b_{g}(x, \bar{x})=\frac{1}{2}
\end{gathered}
$$

and, moreover, $\eta: I \times R \times R \mapsto R$ be defined as

$$
\eta(t, x, \bar{x})=\frac{1}{2}(x-\bar{x}) .
$$

Hence, it can be established by Definition 2.1 , that $f^{i}(t, \cdot, \cdot), i=1,2$, are strictly univex at $\bar{x}$ on $S$ with respect to $\Phi_{f^{i}}, b_{f^{i}}, \eta$ and $\bar{\xi}(t)^{T} g(t, \cdot, \cdot)$ is univex at $\bar{x}$ on $S$ with respect to $\Phi_{g}, b_{g}, \eta$. Using the approach analyzed in the paper, we construct the following multiobjective variational problem $\left(\mathrm{MVP}_{\eta}(\bar{x})\right)$ with the modified objective function as follows:

$$
\begin{aligned}
V \text {-Minimize } & \left(\int_{0}^{1} \frac{1}{4} x \mathrm{~d} t, \int_{0}^{1} x \mathrm{~d} t\right) \\
& \text { subject to } \quad g(t, x, \dot{x})=-x \leqq 0, \quad t \in I \\
& g_{2}(t, x, \dot{x})=x-1 \leqq 0, \quad t \in I, \\
& x(0)=0, x(1)=0 .
\end{aligned}
$$

Since all hypotheses of Theorem 3.1 are fulfilled, we conclude that $\bar{x}(t)=0$ is an efficient solution of the multiobjective variational problem $\left(\operatorname{MVP} 2_{\eta}(\bar{x})\right)$ with the modified objective function. Further, since all hypotheses of Theorem 3.5 are satisfied at $\bar{x}=0$, which is an efficient solution of the multiobjective variational problem $\left(\operatorname{MVP} 2{ }_{\eta}(\bar{x})\right), \bar{x}$ is also efficient in the original multiobjective variational problem (MVP2). Although 
we consider the nonlinear nonconvex multiobjective variational problem (MVP2), then the multiobjective variational problem $\left(\operatorname{MVP}_{\eta}(\bar{x})\right)$ with the modified objective function constructed in the used approach is linear. Then, the multiobjective variational problem $\left(\mathrm{MVP}_{\eta}(\bar{x})\right)$ with the modified objective function is less complex than the original multiobjective variational problem (MVP2). Therefore, it is easier to solve than the original one. This property of the modified objective function method is important from a practical point of view.

\section{Conclusion}

In this paper, the class of nonconvex multiobjective variational programming problems with univex functions has been considered. To solve such nonconvex multiobjective variational programming problems, we have used the modified objective function method. In this method, for the considered multiobjective variational programming problem, its associated multiobjective variational programming problem with the modified objective function has been constructed at the given feasible solution. Under univexity hypotheses, the equivalence between an (weakly efficient) efficient solution of the considered multiobjective variational programming problem and an (weakly efficient) efficient solution of its associated multiobjective variational programming problem with the modified objective function has been established. It turned out that, in general, the multiobjective variational programming problem with the modified objective function generated in the modified objective function method has simpler form than the considered multiobjective variational programming problem. What is more, in some cases, the multiobjective variational programming problem with the modified objective function generated in the modified objective function method is linear or convex although the considered multiobjective variational programming problem is nonlinear and nonconvex. An example of such a nonlinear nonconvex multiobjective variational programming problem has been presented in the paper. The property mentioned above is important from the practical point of view since we are in a position to solve nonlinear nonconvex multiobjective variational programming problems by the help of linear or convex vector variational ones.

Open Access This article is distributed under the terms of the Creative Commons Attribution 4.0 International License (http://creativecommons.org/licenses/by/4.0/), which permits unrestricted use, distribution, and reproduction in any medium, provided you give appropriate credit to the original author(s) and the source, provide a link to the Creative Commons license, and indicate if changes were made.

\section{References}

1. Aghezzaf, B., Khazafi, K.: Sufficient optimality conditions and duality in multiobjective variational programming problems with generalized $b$-invexity. J. Control Cybernet. 33, 1-14 (2004)

2. Ahmad, I., Gualti, T.R.: Mixed type duality for multiobjective variational problems with generalized $(F, \rho)$-convexity. J. Math. Anal. Appl. 306, 669-683 (2005)

3. Antczak, T.: A new approach to multiobjective programming with a modified objective function. J. Global Optim. 27, 485-495 (2003) 
4. Antczak, T.: On efficiency and mixed duality for a new class of nonconvex multiobjective variational control problems. J. Glob. Optim. 59, 757-785 (2014)

5. Antczak, T., Arana-Jiménez, M.: Sufficient optimality criteria and duality for multiobjective variational control problems with $B$-( $p, r)$-invex functions. Opuscula Math. 34, 665-682 (2014)

6. Arana-Jiménez, M., Ruiz-Garzón, G., Rufián-Lizana, A., Osuna-Gómez, R.: A necessary and sufficient condition for duality in multiobjective variational problems. Eur. J. Oper. Res. 201, 672-681 (2010)

7. Bector, C.R., Chandra, S., Gupta, S., Suneja, S.K.: Univex sets, functions and univex nonlinear programming. In: Komlosi, S., Rapcsak, T., Schaible, S. (eds.) Proceedings of Conference of Generalized Convexity, Pecs, Hungary, pp.1-11, Springer (1993)

8. Arana-Jiménez, M., Ruiz-Garzón, G., Rufián-Lizana, A., Osuna-Gómez, R.: Weak efficiency in multiobjective variational problems under generalized convexity. J. Glob. Optim. 52, 109-121 (2012)

9. Arana-Jiménez, M., Ruiz-Garzón, G., Rufián-Lizana, A., Osuna-Gómez, R.: A necessary and sufficient condition for duality in multiobjective variational problems, European. J Oper. Res. 201, 672-681 (2010)

10. Bector, C.R., Husain, I.: Duality for multiobjective variational problems. J. Math. Anal. Appl. 166, 214-229 (1992)

11. Bhatia, D., Kumar, P.: Multiobjective control problem with generalized invexity. J. Math. Anal. Appl. 189, 676-692 (1995)

12. Bhatia, D., Mehra, A.: Optimality conditions and duality for multiobjective variational problems with generalized $B$-invexity. J. Math. Anal. Appl. 234, 341-360 (1999)

13. Craven, B.D.: On continuous programming with generalized convexity. Asia-Pac. J. Oper. Res. 10, 219-232 (1993)

14. Gramatovici, S.: Optimality conditions in multiobjective control problems with generalized invexity. Ann. Univ. Craiova Math. Comp. Sci. Ser. 32, 150-157 (2005)

15. Hachimi, M., Aghezzaf, B.: Sufficiency and duality in multiobjective variational problems with generalized type I functions. J. Glob. Optim. 34, 191-218 (2006)

16. Khazafi, K., Rueda, N.: Multiobjective variational programming under generalized type I univexity. J. Optim. Theory Appl. 142, 363-376 (2009)

17. Khazafi, K., Rueda, N., Enflo, P.: Sufficiency and duality for multiobjective control problems under generalized $(B, \rho)$-type I functions. J. Glob. Optim. 46, 111-132 (2010)

18. Kim, D.S., Kim, M.H.: Generalized type I invexity and duality in multiobjective variational problems. J. Math. Anal. Appl. 307, 533-554 (2005)

19. Mishra, S.K., Wang, S.Y., Lai, K.K.: Generalized Convexity and Vector Optimization, Nonconvex Optimization and Applications, vol. 90. Springer, Berlin, Heidelberg (2009)

20. Mititelu, S.., Postolache, M.: Mond-Weir dualities with Lagrangians for multiobjective fractional and non-fractional variational problems. J. Adv. Math. Stud. 3, 41-58 (2010)

21. Mukherjee, R.N., Mishra, S.K.: Generalized invexity and duality in multiobjective objective variational problems. J. Math. Anal. Appl. 195, 307-322 (1995)

22. Mukherjee, R.N., Mishra, S.K.: Multiobjective control problem with $V$-invexity. J. Math. Anal. Appl. 235, 1-12 (1999)

23. Mukherjee, R.N., Rao, C.P.: Mixed type duality for multiobjective variational problems. J. Math. Anal. Appl. 252, 571-586 (2000)

24. Nahak, C., Nanda, S.: Duality for multiobjective variational problems with invexity. Optimization 36, 235-248 (1996)

25. Nahak, C., Nanda, S.: On efficiency and duality for multiobjective variational control problems with $(F, \rho)$-convexity. J. Math. Anal. Appl. 209, 415-434 (1997)

26. Nahak, C., Behera, N.: Optimality conditions and duality for multiobjective variational problems with generalized $\rho$ - $\eta, \theta)$ - $B$-type-I functions. J. Control Sci. Eng. 2011 (2011). https://doi.org/10.1155/ 2011/497376

27. Reddy, L.V., Mukherjee, R.N.: Efficiency and duality of multiobjective fractional control problems under $(F, \rho)$-convexity. Indian J. Pure Appl. Math. 30, 51-69 (1999)

28. Xiuhong, Ch.: Duality for a class of multiobjective control problems. J. Math. Anal. Appl. 267, 377-394 (2002)

29. Zhian, L., Qingkai, Y.: Duality for a class of multiobjective control problems with generalized invexity. J. Math. Anal. Appl. 256, 446-461 (2001) 